ApJ LetTers ACCEPted For PUbliCATIOn 2012 April 11 (Submitted 2012 MARCH 13).

Preprint typeset using LTEX style emulateapj v. 5/2/11

\title{
DRAMATIC INFRARED VARIABILITY OF WISE J1810-3305: CATCHING EARLY-TIME DUST EJECTION DURING THE THERMAL PULSE OF AN AGB STAR?
}

\author{
Poshak GAndhi, Issei Yamamura And SATOShi TAKita \\ Institute of Space and Astronautical Science, Japan Aerospace Exploration Agency, 3-1-1 Yoshinodai, chuo-ku, Sagamihara, Kanagawa 252-5210, Japan \\ ApJ Letters accepted for publication 2012 April 11 (Submitted 2012 March 13).
}

\begin{abstract}
We present the discovery of a source with broadband infrared photometric characteristics similar to Sakurai's Object. WISE J180956.27-330500.2 (hereafter, J1810-3305) shows very red WISE colors, but a very blue 2MASS [K] vs. WISE [W1 $(3.4 \mu \mathrm{m})$ ] color. It was not visible during the IRAS era, but now has a $12 \mu \mathrm{m}$ flux well above the IRAS point source catalog detection limit. There are also indications of variability in historical optical photographic plates, as well as in multi-epoch AKARI mid-infrared measurements. The broadband infrared spectral energy distribution shape, post-IRAS brightening and multiwavelength variability are all characteristics also shared by Sakurai's Object - a post asymptotic giant branch (post-AGB) star which underwent a late thermal pulse and recently ejected massive envelopes of dust that are currently expanding and cooling. Optical progenitor colors suggest that J1810-3305 may have been of late spectral class. Its dramatic infrared brightening, and the detection of a late-type optical counterpart are consistent with a scenario in which we have caught an extremely massive dust ejection event (in 1998 or shortly before) during the thermal pulse of an AGB star, thus providing a unique opportunity to observe stellar evolution in this phase. J1810-3305 is the only source in the entire WISE preliminary data release with similar infrared SED and variability, emphasizing the rarity of such sources. Confirmation of its nature is of great importance.

Subject headings: stars: AGB and post-AGB — infrared: stars — planetary nebulae: general — stars: individual (Sakurai's Object, WISE J180956.27-330500.2, WISE J1810-3305)
\end{abstract}

\section{INTRODUCTION}

A substantial fraction of cosmic dust is manufactured in late-type stars in the asymptotic giant branch (AGB) phase. Dust and gas are ejected into the interstellar medium at a rate depending upon stellar mass and varying along its evolutionary track (e.g., Vassiliadis \& Wood 1993). For moderate-mass stars $\left(\gtrsim 3 \mathrm{M}_{\odot}\right)$, mass-loss is thought to be quasi-continuous and to evolve gradually, whereas low-mass stars $\left(\sim 1 \mathrm{M}_{\odot}\right)$ experience several explosive mass-loss events triggered by a Helium-burning 'thermal pulse' (e.g., Schroeder et al. 1998). Remnants of such episodic shedding have been observed as detached circumstellar shells in radio observations (e.g., Olofsson et al. 2000, Yamamura et al. 1993) and the infrared (Kerschbaum et al. 2010; Izumiura et al. 2011). But catching ejection events at an early stage is rare given their brevity.

One source which has provided a 'real-time' view of stellar evolution, dust formation and ejection is the so-called born-again post-AGB star, Sakurai's Object (hereafter, 'Sakurai'; Duerbeck \& Benetti 1996). In this class of objects, a white dwarf undergoes a Helium-burning flash resulting in a rejuvenation of the giant phase (Iben et al. 1983), manifesting as drastic changes in broadband spectral shape and intensity on timescales of days-months (Asplund et al. 1999; Haiduk et al. 2005). In the late nineties, Sakurai ejected a massive dust envelope which changed the source optical and infrared appearance completely, and which continues to evolve presently (Kerber et al. 1999b; Käufl et al. 2003; Evans et al. 2006; Chesneau et al. 2009).

Here, we present the discovery of similar massive dust ejection in a previously-unremarkable system, which appears to have been a late-type AGB star. This may be the first time that the early stage of a thermal pulse has been caught in this phase. The finding was made possible by combining new, wide-area infrared sky surveys.

\section{DATA}

\subsection{WISE}

The Wide-field Infrared Survey Explorer (WISE) satellite Wright et al. 2010) has carried out a highly-sensitive all-sky survey in four bands (W1-W4 centered on wavelengths of $\approx 3.4,4.6,12$ and $22 \mu \mathrm{m}$, respectively). The effective angular resolution corresponds to a Gaussian with full-width-athalf-maximum $\approx 6^{\prime \prime}$ in $\mathrm{W} 1-\mathrm{W} 3$, and $12^{\prime \prime}$ in W4. The whole sky was surveyed once between 2010 January and July. Data for about 57\% of the sky were publicly available as of 2012 February, and we used this preliminary data release] in order to select sources. The release includes calibrated Vega source magnitudes. Sources brighter than mags of 8.0, 6.7, 3.8 and -0.4 in W1-4, respectively, begin to be affected by saturation. Profile-fitting is done using only unsaturated pixels, thus extending the dynamic range for point source brightness characterization into the saturated regime 2 . Magnitudes were converted to fluxes using standard zeropoints and color correction assuming a $B_{\nu}(283)$ SED relevant for the sources herein (Table 1 of Wright et al. 2010).

\subsection{Supporting data}

The Two Micron All Sky Survey (2MASS) mapped the sky in the near-IR $J, H$ and $K_{s}$ (hereafter, $K$ ) bands between 1997-2001 from ground-based telescopes at Mt. Hopkins, Arizona and Cerro Tololo, Chile (Skrutskie et al. 2006). We used zeropoints from Cohen et al. (2003).

The InfraRed Astronomy Satellite (IRAS) carried out a pioneering all-sky survey at 12, 25, 60, and $100 \mu \mathrm{m}$ in 1983 (Soifer et al. 1987). A point source catalog (PSC) was produced which covered $96 \%$ of the sky to approximate detec-

\footnotetext{
${ }^{1}$ http://wise2.ipac.caltech.edu/docs/release/prelim/

2 http://wise2.ipac.caltech.edu/docs/release/prelim/expsup/sec4_5c.html
} 
tion limits of $0.5,0.5,0.5$ and $1.5 \mathrm{Jy}$, respectively, in the four bands.

The Japanese mission AKARI possessed two instruments: the InfraRed Camera (IRC; Onaka et al. 2007) and the FarInfrared Surveyor (FIS; Kawada et al. 2007). Its main product was an all-sky catalog covering the IRC 9 and $18 \mu \mathrm{m}$ bands (Ishihara et al. 2010) and FIS 65, 90, 140, and $160 \mu \mathrm{m}$ bands (Yamamura et al. 2010), to $80 \%$ completeness flux limits of 0.12 and $0.22 \mathrm{Jy}$ (IRC) and 3.3, 0.43, 3.6, and 8.2 Jy (FIS), in the respective bands. Its survey lasted from 2006 May to 2007 August. We only used fluxes with a reported quality flag fqual $=3$, indicating good photometric reliability.

Optical photographic plate data and their digitized versions are available over the sky. These date over the $\sim 1970$ s-1990s. More details are given in $\S 4.1$

\section{SAMPLE SELECTION}

During a study of sources with extremely-red WISE colors, we serendipitously found an intriguing source with red WISE colors but a very blue $2 \mathrm{MASS} K$ vs. WISE W1 color, and no IRAS counterpart. In order to investigate this further, we refined and applied the following selection criteria to the entire preliminary data release:

1. $\exists$ a 2 MASS $(K)$ association within $3^{\prime \prime}$ of the WISE position.

2. $K-\mathrm{W} 1<-3.7$.

3. $\mathrm{W} 1-\mathrm{W} 2>3.0$.

4. ph_qual='AAAA'.

5. $\nexists$ an IRAS PSC counterpart within $1^{\prime}$.

The first two criteria select sources with an apparent 2MASS $K$-band flux (in $\nu F_{\nu}$ units) higher than that in WISE $\mathrm{W} 1$ by at least 100 , either due to an intrinsically blue spectral slope, or due to variability between the 2MASS and WISE observation epochs.

The third criterion selects sources with WISE colors characteristic of a cold or reddened SED. This disfavors sources with an intrinsically blue slope.

ph_qual in the fourth criterion is a quality flag assuring secure photometry in all WISE bands (signal-to-noise $\mathrm{S} / \mathrm{N}>10$ ), so that we may explore SED characteristics in detail.

The last criterion favors newly-brightened sources or those without published identifications.

\section{RESULTS}

Of $\sim 257$ million objects in the preliminary data release, only 38 satisfy the WISE+2MASS criteria 1-4 above. These are mostly stars of late (KM) spectral type along with a handful of Mira Cet stars and Carbon stars. Examining our final selection criterion, i.e. the presence of an IRAS PSC counterpart, it turns out that two sources stand apart from the others. These are Sakurai and a source designated as WISE J180956.27-330500.2 (hereafter, 'J1810-3305'). These are the only two sources without an IRAS PSC counterpart. A comparison of the WISE and IRAS fluxes is plotted in Fig. 1 (interestingly, these two sources also stand out in terms of their red W3-W4 color, as shown). Assuming a conservative PSC flux limit of $F_{I R A S}^{\operatorname{limit}}=0.5 \mathrm{Jy}$ and neglecting small color corrections in the IRAS $12 \mu \mathrm{m}$ vs. WISE W3 bandpasses, the above non-detections imply a brightening in the post-IRAS $12 \mu \mathrm{m}$ fluxes by factors of at least 50 (Sakurai) and 13 (J1810-3305), respectively. The W3 fluxes of both lie well above the IRAS PSC limit, and neither is confused with other objects of similar magnitude within a few arcmin, confirmed by careful inspection of the colors and fluxes of neighboring sources. Thumbnail images for J1810-3305 are shown in Fig. 2,

The infrared SEDs of both objects are plotted in Fig. 3, including also $A K A R I$ data. Photometry in the various bands is listed in Table 1. The deep 'dip' at W1 in the SEDs of both sources (according to the selection of mismatched 2MASS and WISE colors) is apparent.

\subsection{Optical counterpart}

Table 1 lists magnitudes of the optical counterpart of J1810-3305 drawn from historical photographic surveys in $B V R I$. These must be used with caution, given that faint object measurement on old plates is subject to increased uncertainty as compared to CCDs. We preferred modern digitized scans where available 3 . Furthermore, the exact epoch of observation is not always explicitly stated in the publicly available data. Magnitudes were converted to approximate fluxes using generic zeropoint 4 for plotting in Fig. 3 Exact bandpass responses are not always available, so these should be considered approximate only.

\subsection{Multiwavelength variability}

Despite the above caveats, there are indications of strong multiwavelength optical variability. J1810-3305 appears to have brightened by $\sim 2$ mags over the approximate period of 1968-1984 (exact dates unknown) in $B$ and $\sim 1$ mag over the 1980 s in $R$. The two available photometric data points in $V$ instead imply fading by $\sim 1$ mag over $\sim 20$ years preceding 1987.

AKARI observed the source over two epochs. The 9 and $18 \mu \mathrm{m}$ fluxes of J1810-3305 declined from 5.6 to $5.0 \mathrm{Jy}$ and from 9.6 to $9.0 \mathrm{Jy}$, respectively, between 2006 September and 2007 March. Typical detection $\mathrm{S} / \mathrm{N} \geq 750$, so these changes are significant.

The source position does not appear to have been covered 5 with other missions, e.g. Spitzer, ISO, Herschel, or HST.

\section{DISCUSSION}

\subsection{Comparison of J1810-3305 and Sakurai}

The similarity in the broadband infrared properties and variability of J1810-3305 and Sakurai is striking. In particular, the 2MASS+WISE SEDs appear to mirror each other well, including the red WISE colors and the apparent W1 dip. Furthermore, both sources have only recently emerged in the midIR. As illustrated by Fig. 1, these properties are unique to J1810-3305 and Sakurai. The nature of J1810-3305 is completely unknown, but these facts suggest that its IR photometric properties are analogous to Sakurai.

Sakurai is known to have ejected a large amount of dust around 1998 (e.g. Geballe et al. 2002). If J1810-3305 also ejected dust recently, the optical and near-IR are expected to

\footnotetext{
3 This was true in the $R$ band, where an older USNO-B1.0 measuremen is $R 2=15.98$ (Monet et al. 2003), about 2 mags fainter than in the digitized sky survey (DSS). We measured source flux in the DSS image and compared it to to field stars, obtaining consistent results with the brighter mag listed in Table 1

${ }^{4}$ http://ssc.spitzer.caltech.edu/warmmission/propkit/pet/magtojy/

${ }^{5}$ Referee communication
} 


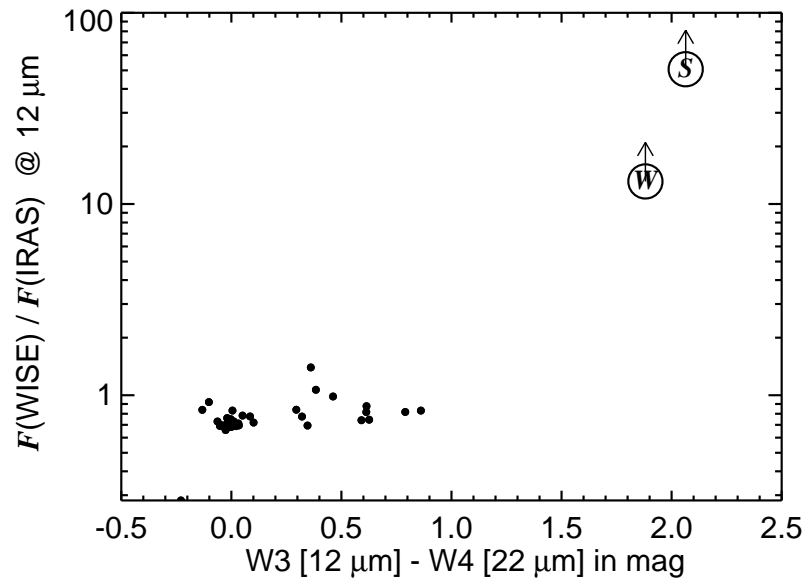

FIG. 1.- Ratio of WISE to IRAS $12 \mu \mathrm{m}$ flux densities vs. WISE color (W3W4) for the 38 sources that satisfy our selection criteria 1-4. J1810-3305 (W) and Sakurai (S) stand out as having brightened dramatically at $12 \mu \mathrm{m}$, and also in terms of their red W3-W4 color.

have faded and reddened since peak brightness, while the mid and far-IR fluxes would rise due to dust reemission. The extremely red W3-W4 color of both these sources in Fig. 1 is indicative of the presence of dust.

The bright near-IR 2MASS fluxes are consistent with hot ( $T \sim 1300 \mathrm{~K}$ ) dust having been freshly ejected in or before 1998 (Fig. 3). Such dust would have been visible with IRAS at $12 \mu \mathrm{m}$ for any modified blackbody with emissivity beta-index $\beta<2.5\left(F_{\nu} \propto \nu^{\beta} B_{\nu, T}\right.$, where $F_{\nu}$ is observed flux density and $B_{\nu, T}$ is the Planck function). This suggests that dust ejection occurred sometime between the IRAS and 2MASS epochs.

The WISE SED implies a much lower thermal temperature $(T \sim 320 \mathrm{~K})$ in 2010 , though a single modified blackbody SED does not fit the observed fluxes well (a mix of multi-temperature components is likely). The apparent 2MASS/WISE 'mismatch' then implies some dust cooling over the $\sim 11+$ interim years.

Integrating over the two blackbody curves in Fig. 3 gives bolometric infrared luminosities $\left(L_{\mathrm{Bol}}\right)$ of $\sim 425 D^{2} \mathrm{~L}_{\odot}$ for the prior 2 MASS component and $\sim 90 D^{2} \mathrm{~L}_{\odot}$ for the recent WISE+AKARI component, where $D$ is the distance in kpc. Unlike Sakurai, where $L_{\mathrm{Bol}}$ has stayed approximately constant, the bolometric IR power of J1810-3305 has declined by a factor of $\sim 5$ between the 2MASS and WISE epochs.

Regarding other wavelengths, in the optical a historical counterpart exists for J1810-3305, but was not known pre1991 for Sakurai. Multiwavelength variability is a common characteristic in both objects. In the far-IR regime, the $A K A R I$ 65 and $90 \mu \mathrm{m}$ data of J1810-3305 lie above the extrapolation of the blackbody curve overlaid on the WISE data (see Fig. 3, suggesting the presence of additional cooler dust already in existence in 2006, or some systematic change in dust properties. Unfortunately, $A K A R I$ did not carry out sufficient scans of Sakurai for accurate far-IR photometry.

\subsection{Extinction}

J1810-3305 lies more than 6 degrees below the Galactic plane $\left(l=-1^{\circ} .0, b=-6^{\circ} .6\right)$. The integrated Galactic neutral gas column $\left(N_{\mathrm{H}}\right)$ along this line-of-sight is determined to be $N_{\mathrm{H}}=1.88 \times 10^{21} \mathrm{~cm}^{-2}$ (Dickey \& Lockman 1990). For a typical Milky Way dust:gas ratio, this translates into a red- dening value of $E_{\mathrm{B}-\mathrm{V}}=0.32$ (Bohlin et al. 1978), or an optical extinction $A_{\mathrm{V}}=1.0$ for a standard extinction curve slope with $R_{\mathrm{V}}=3.1$. Near- and mid-IR extinction is 10-20 times smaller still (see, e.g., several references listed in $\S 2.2 .3$ of Gandhi et al. 2011a). The actual extinction could either be lower if some of the gas column lies behind J1810-3305, or may be somewhat higher if $R_{\mathrm{V}}$ happens to be larger along this line-of-sight. In any case, these numbers suggest that interstellar reddening is unlikely to be extreme, so the SEDs plotted in Fig. 3 should not change dramatically after extinction correction, especially in the IR.

\subsection{Dust mass}

Dust mass may be computed by using the infrared emissivity relation of Hildebrand (1983), given $D$ and the dust characteristics. Here, we simply assume a canonical graphite composition with $0.1 \mu \mathrm{m}$-sized grains (Carbon-rich grains have been identified around Sakurai; Eyres et al. e.g. 1998; Evans et al. e.g. 2006). Specifically, we use Eq. 2 from Gandhi et al. (2011b). For the recent WISE data with $T \sim 320 \mathrm{~K}$, the observed W3 flux is $F_{12 \mu \mathrm{m}}=7.5 \mathrm{Jy}$. This yields a dust mass of $M_{\mathrm{d}}=2.1 \times 10^{-7} D^{2} \mathrm{M}_{\odot}$. If $D=2-$ $5 \mathrm{kpc}$ similar to Sakurai (van Hoof et al. 2007), $M_{\mathrm{d}}=(0.9$ $5) \times 10^{-6} \mathrm{M}_{\odot}$ for $\mathrm{J} 1810-3305$. For a typical gas:dust mass ratio of $\gtrsim 100$, the implied total mass-loss rate is large (cf. Yamamura et al. 1993; Winters et al.2000), suggesting it cannot remain steady. A non-zero $\beta$ index would boost the mass further.

These numbers must be used with caution since the absolute values of dust physical parameters are presently unknown. Instead, a comparison with Sakurai may be useful, for which we find $M_{\text {Sakurai }}(2010$ dust $) \approx 3 \times 10^{-6} D_{\text {Sakurai }}^{2} \mathbf{M}_{\odot}$. Thus, $M_{\mathrm{J} 1810-33} / M_{\text {Sakurai }}=0.07\left(D_{\mathrm{J} 1810-33} / D_{\text {Sakurai }}\right)^{2}$.

\subsection{The nature of J1810-3305}

A major uncertainty is the spectral type of the central star. Assuming a reddening of $E_{\mathrm{B}-\mathrm{V}}=0.3, B-V$ varies from $\approx 2.4$ to $-0.6, V-R$ is about $1-2$ and $R-I \approx 0.5$ or 1.5 , where color measurements are based upon photometry closest matched in time. Uncertainties are $\approx 0.7-1 \mathrm{mag}$. Photometric variability and other caveats mentioned in $\$ 4.1$ and $\S 4.2$ complicate the issue. Fig. 3 plots the plausible range of observed optical fluxes after accounting for these uncertainties. The overall SED shape in the optical appears to have been red, and the redder colors suggest a tentative late ( $\mathrm{K}$ or $\mathrm{M})$ type classification. J1810-3305 may thus be in an earlier evolutionary phase as compared to the post-AGB phase that Sakurai was discovered in.

Sakurai displayed a sharp increase in its optical fluxes around 1991 (Duerbeck \& Benetti 1996). The rising optical phase for J1810-3305 may have already begun before the mid-1980s, according to its brightening $B$ and $R$ magnitudes. If the historical optical data probe pre-dust-ejection photospheric fluxes of a late-type central star with an SED peak around $\sim 1 \mu \mathrm{m}$, then $L_{\mathrm{Bol}}$ should have brightened by $\sim 100$ in order to power the hot 2MASS emission (Fig. 3).

The emerging picture is that we could be observing early dust ejection during the thermal pulse of an AGB star. The presence of an optical pre-cursor, its (tentative) late-type classification, the rapid and strong increase in $L_{\mathrm{Bol}}$ and the massive amount of dust ejected $\left(M_{\mathrm{d}} \sim 10^{-6} \mathrm{M}_{\odot}\right)$ are all consistent with this scenario. The dust ejection epoch $\left(t_{0}\right)$ lies between the IRAS (1983) and 2MASS (1998) observations. 


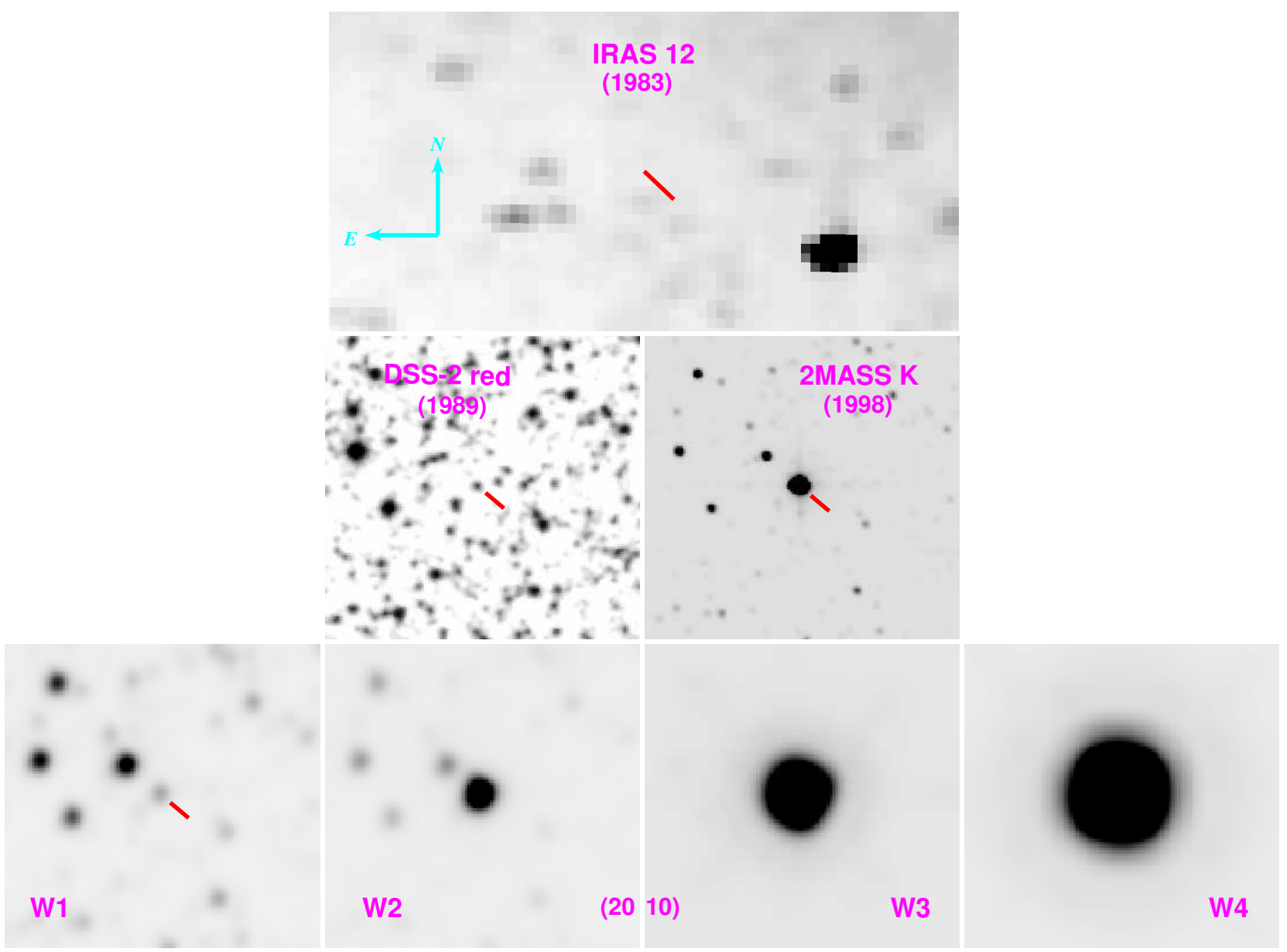

FIG. 2.-IRAS (12 $\mu \mathrm{m}$ ), DSS2 (red), 2MASS (K) and WISE (W1-W4) images of the field of WISE J1810-3305. All images are centered on the source position (marked by a red dash in some). The IRAS image is $100^{\prime} \times 50^{\prime}$ wide; the rest are $2^{\prime} .5$ wide each.

$t_{0}$ may be estimated by assuming that the dust exists in a single shell in instantaneous thermal equilibrium with stellar heating. Based upon the approximate $L_{\mathrm{Bol}}$ and $T$ values derived in $\S 5.1$ the shell radius $r \propto L_{\mathrm{Bol}}{ }^{0.5} T^{-2}$, implying $r_{(W I S E)}=7.6 \times r_{(2 \mathrm{MASS})}$. This expansion took 11.7 years. If the expansion velocity remained constant since $t_{0}$, backward extrapolation places $t_{0}$ between 1996 October and 1998 July. Using a more realistic $\beta$ increases $r_{(W I S E)} / r_{(2 \mathrm{MASS})}$ and pushes $t_{0}$ closer to the 2 MASS epoch.

The $V$ band, on the other hand, shows a mild historical decline. This may be a sign of erratic (early-time) photometric variability such as Sakurai exhibited at late times (Duerbeck et al. 2000), though the large photometric uncertainties make conclusive inferences impossible. If $L_{\mathrm{Bol}}$ did increase $\sim 100$-fold post-1983, it would imply a peak $V$ mag $\sim 10.5-11.4$, similar to Sakurai. If so, why was the appearance of a new bright star not reported for J1810-3305? One possibility is a very brief period between brightening and dust obscuration. Moreover, the source location is far enough outside the Galactic plane that it does not fall in intensively-monitored fields, but is still close enough to the plane that the field is relatively crowded in the optical making it a non-trivial study for amateur observations.

The rapid evolution of Sakurai outpaces all canonical predictions made by theory of post-AGB stars, which expects slower flux changes from the onset of the Helium flash (Iben et al. 1983; Kerber et al. 1999a; Herwig 2001). In con- trast, J1810-3305 may now be providing us a testbed for studying the Helium flash of the (earlier) AGB phase. The infrared brightening and reddening appear to be coeval in both objects, but the earlier optical rise of J1810-3305 suggests that the optical evolution of J1810-3305 may be slightly slower than that of Sakurai $(\S 4.1$. Furthermore, the post2MASS $L_{\text {Bol }}$ decline indicates that the central star could already be relaxing following the thermal pulse, unlike the apparently steady output of Sakurai. If so, the thermal pulse lasted no more than $\sim 30$ years - the interval from the optical measurements of the late-1980s to the WISE epoch. This is exceptionally-brief even compared to the 'ultra-short' massloss timescales of $\sim 10^{2}$ years considered in the literature (e.g. Iben 1982; Schroeder et al. 1998). The other possibility is that multi-temperature expanding shells result in complex optical depth effects unaccounted for in the $L_{\mathrm{Bol}}$ computation.

Large sky surveys are optimal for serendipitously catching rare objects. The fact that only two sources (J1810-3305 and Sakurai) in the entire WISE preliminary database share similar properties emphasizes the rare (brief) nature of such events. Only a handful of other sources have been compared to Sakurai (e.g. A 30, A 78, FG Sge, V605 Aql and V838 Mon; Jacoby \& Ford 1983; Lawlor 2005; Guerrero et al. 2012). Almost all are in more advanced states of evolution, and none appears to be coeval with Sakurai. J1810-3305 thus provides a unique opportunity for follow-up.

Ascertaining the degree of affinity between these sources 

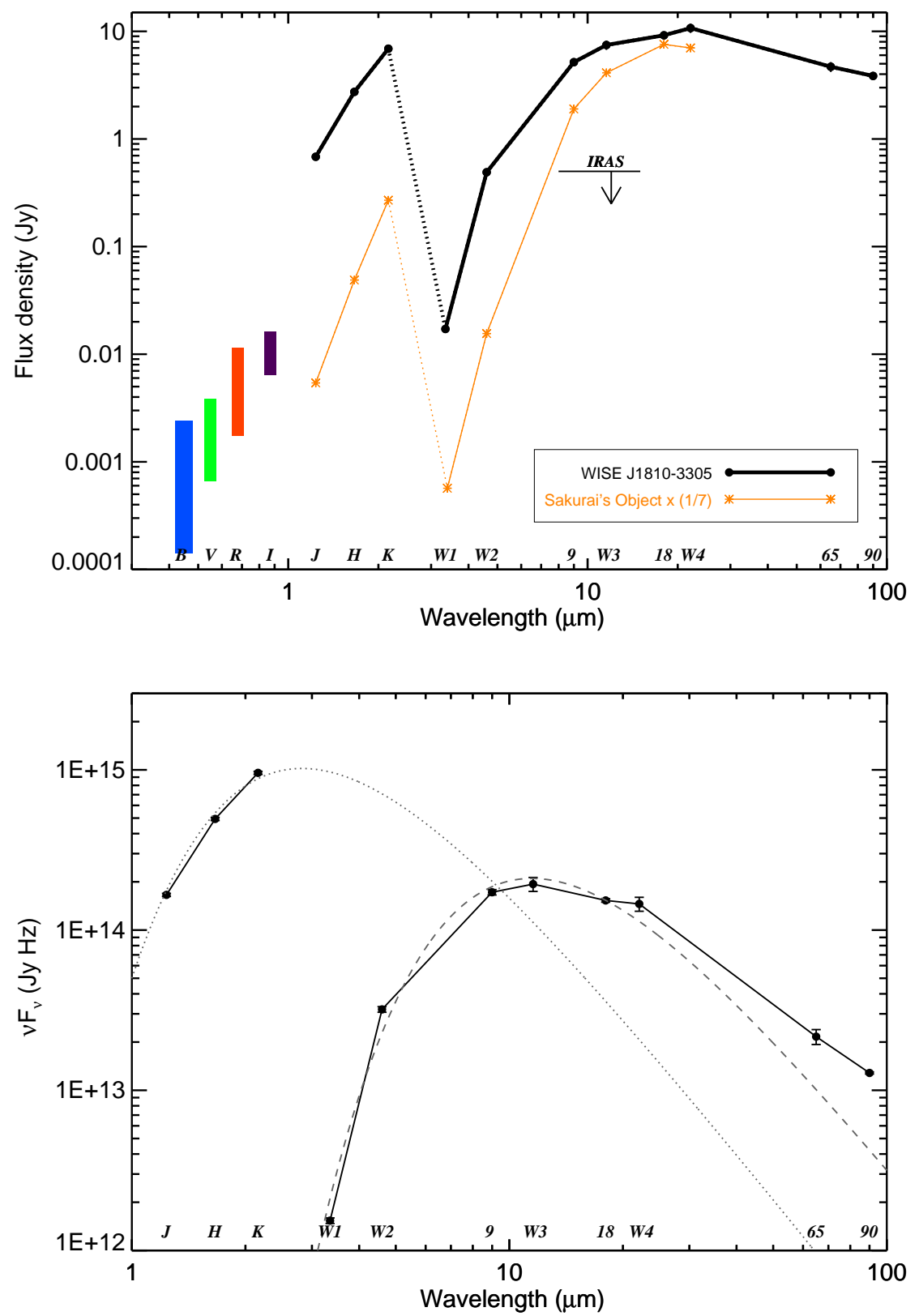

FIG. 3.- (Top) Broadband infrared SED of WISE J1810-3305 (top thick lines). Dotted lines separate older 2MASS data from newer mid-IR (WISE+AKARI) observations. Optical data are denoted by the color bars at the bottom left, and include \pm 0.5 mags to account for variability and systematic photometric calibration uncertainty. The SED of Sakurai's Object, scaled down by a factor of 7 for clarity, is shown as the lower thin (orange) lines. (Bottom) Planck functions overlaid on the IR SED of J1810-3305 in $\nu F_{\nu}$ units. The dotted curve covers the 2MASS points and has a temperature $(T)$ of $1300 \mathrm{~K}$, whereas the dashed curve over the WISE data is for $T=320 \mathrm{~K}$. These models are for illustrative purposes only, in order to emphasize the overall change in $T$, and the $A K A R I$ far-IR excess.

requires optical and infrared spectroscopic observations of J1810-3305 in order to determine its spectral class, measure element abundances and understand the nature of circumstellar dust. Irrespective of these comparisons, however, it appears that we have identified a massive dust ejection event at early times, making WISE J1810-3305 an interesting object in its own right.

PG acknowledges a JAXA International Top Young Fellow- ship, and ST a JSPS Postdoctoral Fellowship. WISE is a project of Univ. California, Los Angeles, and Jet Propulsion Laboratory (JPL)/California Institute of Technology (Caltech), funded by the National Aeronautics and Space Administration (NASA). 2MASS is a project of the Univ. Massachusetts and the Infrared Processing and Analysis Center/Caltech, funded by NASA and the National Science Foundation. $A K A R I$ is a project of the Japan Aerospace Exploration Agency with the participation of the European Space Agency. Data products from the IRAS missions were important for the analysis herein. The NASA/IPAC Infrared Science Archive (IRSA) operated by JPL under contract with NASA, 
and SIMBAD operated at CDS, Strasbourg, France, were the main databases queried. The USNO Image and Catalogue Archive is operated by the U.S. Naval Observatory. The DSS was produced at the Space Telescope Science Institute under
U.S. Government grant NAG W-2166, and the UK Schmidt Telescope operated by the Royal Observatory Edinburgh and by the Anglo-Australian Observatory. The expert referee's review was prompt and valuable.

\section{REFERENCES}

Asplund, M., Lambert, D. L., Kipper, T., Pollacco, D., \& Shetrone, M. D. 1999, A\&A, 343, 507

Bohlin, R. C., Savage, B. D., \& Drake, J. F. 1978, ApJ, 224, 132

Chesneau, O., Clayton, G. C., Lykou, F., de Marco, O., Hummel, C. A., Kerber, F., Lagadec, E., Nordhaus, J., Zijlstra, A. A., \& Evans, A. 2009, A\&A, 493, L17

Cohen, M., Wheaton, W. A., \& Megeath, S. T. 2003, AJ, 126, 1090

Dickey, J. M., \& Lockman, F. J. 1990, ARA\&A, 28, 215

Duerbeck, H. W., \& Benetti, S. 1996, ApJL, 468, L111

Duerbeck, H. W., Liller, W., Sterken, C., Benetti, S., van Genderen, A. M., Arts, J., Kurk, J. D., Janson, M., Voskes, T., Brogt, E., Arentoft, T., van der Meer, A., \& Dijkstra, R. 2000, AJ, 119, 2360

Evans, A., Tyne, V. H., van Loon, J. T., Smalley, B., Geballe, T. R., Gehrz, R. D., Woodward, C. E., Zijlstra, A. A., Polomski, E., Rushton, M. T., Eyres, S. P. S., Starrfield, S. G., Krautter, J., \& Wagner, R. M. 2006, MNRAS, 373, L75

Eyres, S. P. S., Evans, A., Geballe, T. R., Salama, A., \& Smalley, B. 1998, MNRAS, 298, L37

Gandhi, P., Blain, A. W., Russell, D. M., Casella, P., Malzac, J., Corbel, S., D'Avanzo, P., Lewis, F. W., Markoff, S., Cadolle Bel, M., Goldoni, P., Wachter, S., Khangulyan, D., \& Mainzer, A. 2011a, ApJL, 740, L13+

Gandhi, P., Isobe, N., Birkinshaw, M., Worrall, D. M., Sakon, I., Iwasawa, K., \& Bamba, A. 2011b, PASJ, 63, 505

Geballe, T. R., Evans, A., Smalley, B., Tyne, V. H., \& Eyres, S. P. S. 2002, Ap\&SS, 279, 39

Girard, T. M., Platais, I., Kozhurina-Platais, V., van Altena, W. F., \& López, C. E. 1998 , AJ, 115, 855

Guerrero, M. A., Ruiz, N., Hamann, W.-R., Chu, Y.-H., Todt, H., Schoenberner, D., Oskinova, L., Gruendl, R. A., Steffen, M., Blair, W. P., \& Toala, J. A. 2012, ApJ submitted, arXiv:1202.4463

Hajduk, M., Zijlstra, A. A., Herwig, F., van Hoof, P. A. M., Kerber, F., Kimeswenger, S., Pollacco, D. L., Evans, A., Lopéz, J. A., Bryce, M., Eyres, S. P. S., \& Matsuura, M. 2005, Science, 308, 231

Herwig, F. 2001, ApJL, 554, L71

Hildebrand, R. H. 1983, QJRAS, 24, 267

Iben, Jr., I. 1982, ApJ, 260, 821

Iben, Jr., I., Kaler, J. B., Truran, J. W., \& Renzini, A. 1983, ApJ, 264, 605 Ishihara, D., Onaka, T., Kataza, H., Salama, A., Alfageme, C., Cassatella, A., Cox, N., García-Lario, P., Stephenson, C., Cohen, M., Fujishiro, N., Fujiwara, H., Hasegawa, S., Ita, Y., Kim, W., Matsuhara, H., Murakami, H., Müller, T. G., Nakagawa, T., Ohyama, Y., Oyabu, S., Pyo, J., Sakon, I., Shibai, H., Takita, S., Tanabé, T., Uemizu, K., Ueno, M., Usui, F., Wada, T., Watarai, H., Yamamura, I., \& Yamauchi, C. 2010, A\&A, 514, A1 Izumiura, H., Ueta, T., Yamamura, I., Matsunaga, N., Ita, Y., Matsuura, M., Nakada, Y., Fukushi, H., Mito, H., Tanabé, T., \& Hashimoto, O. 2011, A\&A, 528, A29

Jacoby, G. H., \& Ford, H. C. 1983, ApJ, 266, 298

Käufl, H. U., Koller, J., \& Kerber, F. 2003, A\&A, 406, 981
Kawada, M., Baba, H., Barthel, P. D., Clements, D., Cohen, M., Doi, Y., Figueredo, E., Fujiwara, M., Goto, T., Hasegawa, S., Hibi, Y., Hirao, T., Hiromoto, N., Jeong, W.-S., Kaneda, H., Kawai, T., Kawamura, A., Kester, D., Kii, T., Kobayashi, H., Kwon, S. M., Lee, H. M., Makiuti, S. Matsuo, H., Matsuura, S., Müller, T. G., Murakami, N., Nagata, H., Nakagawa, T., Narita, M., Noda, M., Oh, S. H., Okada, Y., Okuda, H., Oliver, S., Ootsubo, T., Pak, S., Park, Y.-S., Pearson, C. P.,

Rowan-Robinson, M., Saito, T., Salama, A., Sato, S., Savage, R. S., Serjeant, S., Shibai, H., Shirahata, M., Sohn, J., Suzuki, T., Takagi, T., Takahashi, H., Thomson, M., Usui, F., Verdugo, E., Watabe, T., White, G. J., Wang, L., Yamamura, I., Yamauchi, C., \& Yasuda, A. 2007, PASJ, 59,389

Kerber, F., Blommaert, J. A. D. L., Kimeswenger, S., Groenewegen, M. A. T., \& Kaufl, H. U. 1999a, in ESA Special Publication, Vol. 427, The Universe as Seen by ISO, ed. P. Cox \& M. Kessler, 357

Kerber, F., Köppen, J., Roth, M., \& Trager, S. C. 1999b, A\&A, 344, L79

Kerschbaum, F., Ladjal, D., Ottensamer, R., Groenewegen, M. A. T., Mecina, M., Blommaert, J. A. D. L., Baumann, B., Decin, L., Vandenbussche, B., Waelkens, C., Posch, T., Huygen, E., De Meester, W., Regibo, S., Royer, P., Exter, K., \& Jean, C. 2010, A\&A, 518, L140

Lasker, B. M., Lattanzi, M. G., McLean, B. J., Bucciarelli, B., Drimmel, R., Garcia, J., Greene, G., Guglielmetti, F., Hanley, C., Hawkins, G., Laidler, V. G., Loomis, C., Meakes, M., Mignani, R., Morbidelli, R., Morrison, J., Pannunzio, R., Rosenberg, A., Sarasso, M., Smart, R. L., Spagna, A., Sturch, C. R., Volpicelli, A., White, R. L., Wolfe, D., \& Zacchei, A. 2008, AJ, 136, 735

Lawlor, T. M. 2005, MNRAS, 361, 695

Monet, D. G., Levine, S. E., Canzian, B., Ables, H. D., Bird, A. R., Dahn, C. C., Guetter, H. H., Harris, H. C., Henden, A. A., Leggett, S. K., Levison, H. F., Luginbuhl, C. B., Martini, J., Monet, A. K. B., Munn, J. A., Pier, J. R., Rhodes, A. R., Riepe, B., Sell, S., Stone, R. C., Vrba, F. J., Walker, R. L., Westerhout, G., Brucato, R. J., Reid, I. N., Schoening, W., Hartley, M., Read, M. A., \& Tritton, S. B. 2003, AJ, 125, 984

Olofsson, H., Bergman, P., Lucas, R., Eriksson, K., Gustafsson, B., \& Bieging, J. H. 2000, A\&A, 353, 583

Onaka, T., Matsuhara, H., Wada, T., Fujishiro, N., Fujiwara, H., Ishigaki, M., Ishihara, D., Ita, Y., Kataza, H., Kim, W., Matsumoto, T., Murakami, H., Ohyama, Y., Oyabu, S., Sakon, I., Tanabé, T., Takagi, T., Uemizu, K., Ueno, M., Usui, F., Watarai, H., Cohen, M., Enya, K., Ootsubo, T., Pearson, C. P., Takeyama, N., Yamamuro, T., \& Ikeda, Y. 2007, PASJ, 59, 401

Schroeder, K.-P., Winters, J. M., Arndt, T. U., \& Sedlmayr, E. 1998, A\&A, 335, L9

Skrutskie, M. F., Cutri, R. M., Stiening, R., Weinberg, M. D., Schneider, S., Carpenter, J. M., Beichman, C., Capps, R., Chester, T., Elias, J., Huchra, J., Liebert, J., Lonsdale, C., Monet, D. G., Price, S., Seitzer, P., Jarrett, T., Kirkpatrick, J. D., Gizis, J. E., Howard, E., Evans, T., Fowler, J., Fullmer, L., Hurt, R., Light, R., Kopan, E. L., Marsh, K. A., McCallon, H. L., Tam, R., Van Dyk, S., \& Wheelock, S. 2006, AJ, 131, 1163

Soifer, B. T., Neugebauer, G., \& Houck, J. R. 1987, ARA\&A, 25, 187

van Hoof, P. A. M., Hajduk, M., Zijlstra, A. A., Herwig, F., Evans, A., van de Steene, G. C., Kimeswenger, S., Kerber, F., \& Eyres, S. P. S. 2007, A\&A, 471, L9

Vassiliadis, E., \& Wood, P. R. 1993, ApJ, 413, 641

Winters, J. M., Le Bertre, T., Jeong, K. S., Helling, C., \& Sedlmayr, E. 2000, A\&A, 361, 641

Wright, E. L., Eisenhardt, P. R. M., Mainzer, A. K., Ressler, M. E., Cutri, R. M., Jarrett, T., Kirkpatrick, J. D., Padgett, D., McMillan, R. S., Skrutskie, M., Stanford, S. A., Cohen, M., Walker, R. G., Mather, J. C., Leisawitz, D., Gautier, III, T. N., McLean, I., Benford, D., Lonsdale, C. J., Blain, A., Mendez, B., Irace, W. R., Duval, V., Liu, F., Royer, D., Heinrichsen, I., Howard, J., Shannon, M., Kendall, M., Walsh, A. L., Larsen, M., Cardon, J. G., Schick, S., Schwalm, M., Abid, M., Fabinsky, B., Naes, L., \& Tsai, C.-W. 2010, AJ, 140, 1868

Yamamura, I., Makiuti, S., Ikeda, N., Fukuda, Y., Oyabu, S., Koga, T., \& White, G. J. 2010, VizieR Online Data Catalog, 2298, 0

Yamamura, I., Onaka, T., Kamijo, F., Izumiura, H., \& Deguchi, S. 1993, PASJ, 45, 573

Zacharias, N., Monet, D. G., Levine, S. E., Urban, S. E., Gaume, R., \& Wycoff, G. L. 2005, VizieR Online Data Catalog, 1297, 0 
TABLE 1

PHOTOMETRY OF WISE J1810-3305 AND SAKURAI's OBJECT

\begin{tabular}{|c|c|c|c|c|c|c|}
\hline \multirow{3}{*}{ Band } & \multicolumn{3}{|c|}{ J1810-3305 } & \multicolumn{3}{|c|}{ Sakurai's Object } \\
\hline & Epoch & Mag & Flux density & Epoch & Mag & Flux density \\
\hline & & Vega & Jy & & Vega & Jy \\
\hline $\mathrm{B}^{[1]}$ & 1966-74 (1968?) & $18.17 \pm 0.48$ & & & & \\
\hline $\mathrm{B} 2^{[2]}$ & $1978-90$ (1984?) & 16.09 & & & & \\
\hline $\mathrm{V}^{[1]}$ & 1966-74 (1968?) & 15.49 & & & & \\
\hline $\mathrm{V}^{[3]}$ & 1987.710 & $16.40 \pm 0.47$ & & & & \\
\hline $\mathrm{R} 1^{[2]}$ & $1974-87$ (1984.2?) & 15.07 & & & & \\
\hline $\mathrm{R}^{[3]}$ & 1989.677 & $14.03 \pm 0.45$ & & & & \\
\hline$I^{[2]}$ & 1978-2002 (1990?) & $13.36 \pm 0.46$ & & & & \\
\hline 2MASS J & 1998.57 & $8.42 \pm 0.02$ & $0.68 \pm 0.02$ & 1999.33 & $11.56 \pm 0.02$ & $0.037 \pm 0.001$ \\
\hline 2MASS H & 1998.57 & $6.43 \pm 0.02$ & $2.74 \pm 0.06$ & 1999.33 & $8.69 \pm 0.03$ & $0.34 \pm 0.01$ \\
\hline 2MASS K & 1998.57 & $4.96 \pm 0.02$ & $6.90 \pm 0.15$ & 1999.33 & $6.37 \pm 0.02$ & $1.84 \pm 0.04$ \\
\hline AKARI 9 & 2006/09-2007/03 (2006.92) & & $5.17 \pm 0.20$ & 2006/09-2007/03 (2006.97) & & $13.26 \pm 0.65$ \\
\hline AKARI 18 & 2006/09-2007/03 (2006.92) & & $9.19 \pm 0.23$ & $2006 / 09-2007 / 03$ (2006.97) & & $53.01 \pm 0.81$ \\
\hline AKARI 65 & 2006/09-2007/03 (2006.92) & & $4.69 \pm 0.50$ & $\ldots$ & $\ldots$ & ... \\
\hline AKARI 90 & 2006/09-2007/03 (2006.92) & & $3.85 \pm 0.06$ & $\ldots$ & $\ldots$ &. \\
\hline W1 & $2010 / 03(2010.222)$ & $10.27 \pm 0.03$ & $0.017 \pm 0.001$ & $2010 / 03(2010.215)$ & $11.86 \pm 0.07$ & $(4.0 \pm 0.3) \times 10^{-3}$ \\
\hline W2 & $2010 / 03(2010.222)$ & $6.24 \pm 0.02$ & $0.49 \pm 0.01$ & $2010 / 03(2010.215)$ & $7.87 \pm 0.02$ & $0.109 \pm 0.002$ \\
\hline W3 & $2010 / 03(2010.222)$ & $1.62 \pm 0.01$ & $7.47 \pm 0.07$ & $2010 / 03(2010.215)$ & $0.15 \pm 0.003$ & $28.8 \pm 0.1$ \\
\hline W4 & $2010 / 03(2010.222)$ & $-0.27 \pm 0.01$ & $10.7 \pm 0.1$ & $2010 / 03(2010.215)$ & $-1.92 \pm 0.03$ & $49.0 \pm 1.3$ \\
\hline
\end{tabular}

${ }^{[1]}$ NOMAD/YB6/SPM (Bandpasses 103aO and 103aG+OG515 for $B$ and $V$ ); Zacharias et al. (2005), Girard et al. (1998); ${ }^{22]}$ USNO-B1.0: (Surveys: R1:

ESO-R; B2: SERC-J; I: SERC-I); Monet et al. (2003); ${ }^{33}$ DSS+GSC2.3: (XV/V495 and AAO-SES/XS/IIIa-F+OG590 for $V$ and $R$ ); Lasker et al. (2008). For multiple observations (e.g. AKARI/WISE), the median epoch is stated in brackets, and flux is the mean reported value. For historical optical surveys, the exact observation epoch is not always available; uncertain epochs or the median date of the full survey are stated with '?'. For the DSS, the DATE-OBS fits header keyword is stated. Quoted photometric errors, where available, are statistical. 